\title{
ON THE LEBESGUE MEASURABILITY OF CONTINUOUS FUNCTIONS IN CONSTRUCTIVE ANALYSIS
}

\author{
DOUGLAS BRIDGES AND OSVALD DEMUTH
}

\begin{abstract}
The paper opens with a discussion of the distinction between the classical and the constructive notions of "computable function." There then follows a description of the three main varieties of modern constructive mathematics: Bishop's constructive mathematics, the recursive constructive mathematics of the Russian School, and Brouwer's intuitionistic mathematics. The main purpose of the paper is to prove the independence, relative to Bishop's constructive mathematics, of each of the following statements:

There exists a bounded, pointwise continuous map of $[0,1]$ into $\mathbf{R}$ that is not Lebesgue measurable.

If $\mu$ is a positive measure on a locally compact space, then every real-valued map defined on a full set is measurable with respect to $\mu$.
\end{abstract}

The purpose of this article is to answer the following question within a framework which makes the discussion accessible to mathematicians who know little or nothing about the foundational technicalities of modern constructive mathematics:

In constructive mathematics, are there any realvalued functions defined on $[0,1]$ that are not Lebesgue measurable?

As we shall see, the answer to this question depends on the variety of constructive mathematics within which measure theory is developed.

We first explain the difference between constructive mathematics and the "classical" mathematics practised by the majority of our colleagues today, and describe the three varieties of constructive mathematics with which this paper is concerned. The reader

Received by the editors November 6, 1989.

1980 Mathematics Subject Classification (1985 Revision). Primary 03F25, 03F65, 28A20; Secondary 03F55, 03F60.

The second author is deceased. 
may find it helpful to consult Chapters 1 and 2 of Bridges-Richman at this stage.

The main difference between constructive and classical mathematics lies in the information considered sufficient to characterize mathematical objects and their properties. In particular, the constructive mathematician pays close attention to the interpretation of the phrase "there exists:" if he says that there exists an object $x$ with the property $P$, he means that he has an algorithm for constructing, to any desired degree of approximation, a certain object $x$ and for verifying that $x$ has the property $P$; it is not enough for him to establish that the assumption "there does not exist an object $x$ with the property $P$ " leads to a contradiction, as an argument of that sort does not, of itself, provide a construction of the required object $x$.

To illustrate these distinctions, let us consider a comparatively simple mathematical notion: that of a binary sequence. Classically, this is simply a sequence in which each term belongs to $\{0,1\}$; but from our constructive point of view a binary sequence $\left(a_{n}\right)$ is an algorithm which, applied to any positive integer $n$, produces an output $a_{n}$ belonging to $\{0,1\}$.

Note that, in order to justify constructively the assertion that $a_{n}$ belongs to $\{0,1\}$, we must be able to tell whether $a_{n}=0$ or $a_{n}=1$; it does not suffice to prove that $a_{n}$ cannot fail to belong to $\{0,1\}$. Consider, for example, the following putative specification of a binary sequence $\left(f_{n}\right)$ :

$$
\begin{aligned}
f_{n} & =0 \quad \text { if Fermat's conjecture is false } \\
& =1 \quad \text { if Fermat's conjecture is true. }
\end{aligned}
$$

This is an acceptable classical definition of a binary sequence; moreover, traditional computability theory says that $\left(f_{n}\right)$ is a computable binary sequence, since, classically, there exists an algorithm -either the algorithm with constant output 0 , or that with constant output 1 -which, applied to any positive integer $n$, outputs $f_{n}$ [Kfoury et al., p. 2; cf. Rogers, pp. 9-10]. However, the above information does not specify a constructive binary sequence, since, in the absence of a resolution of Fermat's conjecture, we cannot tell which of the two algorithms in question computes the terms $f_{n}$. Of course, if Fermat's conjecture were resolved tomorrow, then we would be able to say which algorithm produced the sequence $\left(f_{n}\right)$, which would then be constructively defined. 
A more dramatic example of the distinction under discussion is provided by the following definition: for all natural numbers $n$,

$$
\begin{aligned}
c_{n} & =0 \quad \text { if the Continuum Hypothesis is true, } \\
& =1 \quad \text { if the Continuum Hypothesis is false. }
\end{aligned}
$$

Here we have a classically acceptable binary sequence which is "computable" by one of two constant-output algorithms, but for which, within Zermelo-Fraenkel Set Theory with the Axiom of Choice, we will never be able to decide which of these two algorithms computes the terms $c_{n}$. Within constructive mathematics, however, $\left(c_{n}\right)$ poses no problem: it is simply not defined constructively.

It is hard to take seriously a definition of "computable binary sequence" which applies to $\left(c_{n}\right)$. We believe that there is a meaningful distinction between sequences like $\left(f_{n}\right)$ and $\left(c_{n}\right)$ on the one hand, and constructive (in our sense) binary sequences on the other. Since classical mathematics does not possess the logical refinement necessary to reveal that distinction, there is a strong-we would argue overwhelming - case for the use of constructive logic in computability theory. ${ }^{1}$

Now consider the following statement, which Bishop has labelled the limited principle of omniscience:

LPO If $\left(a_{n}\right)$ is a binary sequence, then either there exists $n$ such that $a_{n}=1$, or else $a_{n}=0$ for all $n$.

In the classical setting, pure logic dictates that LPO is trivially true. But, interpreted constructively, LPO is a much stronger statement than at first appears: if LPO holds constructively, then there exists an algorithm $\mathscr{A}$ whose input set consists of all binary sequences, whose outputs belong to $\mathbf{N} \cup\{-1\}$, and which, on being applied to a binary sequence $\left(a_{n}\right)$, behaves as follows:

if there exists $n$ with $a_{n}=1$, then $\mathscr{A}$ outputs such a natural number $n$;

$$
\text { if } a_{n}=0 \text { for all } n \text {, then } \mathscr{A} \text { outputs }-1 \text {. }
$$

\footnotetext{
${ }^{1}$ Moreover, by regarding such sequences as $\left(f_{n}\right)$ and $\left(c_{n}\right)$ as "computable," classical computability theory would seem to be divorcing itself from computational practice. What would happen if, in response a request that he write software to perform a certain computation, an employee presented his boss with two programs and the information that, although one of these programs performed the required computation, he did not know which one?
} 
A moment's reflection should suggest to anyone familiar with computer programming that such an algorithm is unlikely ever to materialize, and therefore that LPO should not be accepted as a principle of constructive mathematics. To turn that suggestion into complete conviction, we need only observe that within the context of recursion theory, in which binary sequences are recursive functions with values in $\{0,1\}$, there is no recursive algorithm which, applied to any binary sequence $\left(a_{n}\right)$, will output 1 if there exists an index $n$ with $a_{n}=1$, and output 0 if $a_{n}=0$ for all $n$ : indeed, such an algorithm would provide us with a procedure for deciding, for any program $P$ with integer inputs and any integer $n$, whether or not $P$ will halt when given the input $n$ [BridgesRichman, Chapter 3, (1.2)-(1.4)]. It is a basic and famous result of elementary recursion theory that no such decision procedure exists, even classically [Rogers, Chapter 1, Theorem VII; Kfoury et al., pp. 10, 49].

There are many important results of classical mathematics for which a constructive proof could be transformed into one of LPO, and which are therefore constructively unacceptable. Among the more elementary of such results are the following:

(i) the law of trichotomy for real numbers: for each real number $x, x>0$ or $x=0$ or $x<0$;

(ii) the least-upper-bound principle for sequences: to each sequence $\left(a_{n}\right)$ of real numbers that is bounded above, there corresponds an upper bound $s$ such that for each $\varepsilon>0$ there exists $n$ with $a_{n}>s-\varepsilon$;

(iii) the sequential compactness property of the closed interval $[0,1]$ : each sequence in $[0,1]$ contains a convergent subsequence.

In fact, for slightly different reasons, we do not expect to find a constructive proof of even the following weak form of trichotomy for $\mathbf{R}$ :

(iv) for each real number $x$, either $x \geq 0$ or $x \leq 0$.

Fortunately, there are good constructive substitutes for the inadmissible trichotomy laws. For example, we can prove that if it is impossible that the real number $x$ satisfies $x>0$, then 
$x \leq 0 .^{2}$ Also, if $a, b$ are real numbers with $a<b$, then for any real number $x$ either $a<x$ or $x<b$. The last result is commonly used to split a constructive argument into cases, where a split of the form "either $x>a$ or $x \leq a$ " would be unacceptable.

Since the main object of our paper is an investigation of Lebesgue measurability, it is appropriate to end this brief discussion of the differences between constructive and classical mathematics by pointing out that the failure of LPO to hold constructively has serious repercussions within measure theory: for example, it implies that (whatever the constructive definition of the Lebesgue integral and related concepts may be) Lebesgue's classical monotone convergence theorem will not hold unchanged in the constructive setting. In order to obtain a viable constructive version of Lebesgue's theorem, we are forced to postulate the existence (computability!) of the relevant supremum. To be precise, the constructive theorem states that

If $f_{1} \leq f_{2} \leq \cdots$ are Lebesgue integrable functions on $[0,1]$, then $\left(f_{n}\right)$ converges in measure to a Lebesgue integrable function if and only if the sequence $\left(\int f_{n}\right)$ of integrals converges to a limit in R [Bishop-Bridges, Chapter 6, (8.6)].

\section{2}

We now describe the three varieties of constructive mathematics with which we shall work below. These varieties differ in several respects, not least in the notion of algorithm that they admit.

The first variety, Bishop's constructive mathematics (hereafter referred to as BISH), considers "algorithm" to be a primitive notion, does not depend on any special formalism, and is consistent with each of the other two varieties and with classical mathematics (CLASS); in particular, every proof of a theorem within BISH is also a proof of that theorem in classical mathematics. In view of

\footnotetext{
${ }^{2}$ Note that the statement $\forall x \in \mathbf{R}(\neg(x \leq 0) \Rightarrow x>0)$ is constructively equivalent to Markov's principle,

If $\left(a_{n}\right)$ is a binary sequence such that $\neg \forall n\left(a_{n}=0\right)$, then there exists $n$ such that $a_{n}=1$,

which embodies an unbounded search. Although Markov's principle is accepted, with some reservations, by practitioners of the Russian School of recursive constructive mathematics, it is not accepted by most constructive mathematicians. We shall not use it in this paper.
} 
this consistency, BISH can be regarded as the constructive core of classical mathematics.

The second variety, the constructive mathematics of the Russian School of Markov (RUSS), is based on the same underlying logical principles as BISH, but pins down the notion of algorithm by adopting the Church-Markov-Turing thesis that every sequence of natural numbers is recursive. It also studies only those mathematical objects which are effectively codable by natural numbers. (For example, Gödel numbers are effective codes of partial recursive functions.) Thus, in a highly consistent way, the practitioners of RUSS endorse Bishop's view that

The primary concern of mathematics is number, and this means the positive integers ... Everything attaches itself to number, and every mathematical statement ultimately expresses the fact that if we perform certain computations within the set of positive integers, we shall obtain certain results. [Bishop, pp. 2-3].

It is important to note that although RUSS contains results that at first sight contradict theorems of classical mathematics, if such results are correctly interpreted they are acceptable within the classical system. For example, there exists in RUSS a pointwise continuous function $f:[0,1] \rightarrow \mathbf{R}$ that is not uniformly continuous. However, this does not contradict the classical uniform continuity theorem: from a classical point of view, $f$ is a recursive function defined and recursively pointwise continuous on the set of recursive real numbers; but, precisely because of the uniform continuity theorem, $f$ cannot be extended to a pointwise continuous function on the set of all classical real numbers (recursive and nonrecursive) between 0 and 1 inclusive.

The third variety, and historically the oldest, is Brouwer's intuitionistic mathematics (INT). Based on Brouwer's intuitionistic philosophy, this variety uses a classically inexplicable notion of "free choice sequence" to develop principles that lead to results apparently inconsistent with both classical mathematics and RUSS [Heyting]. In this case, it is more appropriate to say that these results, and the intuitionistic constructions used to obtain them, cannot be interpreted using the philosophy and methods of classical mathematics.

If, at the risk of offending strict adherents of RUSS or INT, we work formally and forget the underlying philosophies, each of 
these two varieties can be viewed as BISH with certain adjoined principles. In the case of RUSS, the essential principle adjoined to BISH is the Church-Markov-Turing thesis, although another principle, due to Markov, is also added in some contexts (see, for example, Bridges-Richman, Chapter 2, (6.5)). From our point of view, the most significant consequence of the Church-MarkovTuring thesis is a singular covering property of the line which embodies a strong denial of the Heine-Borel theorem; this property will be explained in detail shortly.

On the other hand, INT can be viewed formally as BISH together with certain principles which lead to the intuitionistic uniform continuity theorem:

UC Every function from a compact metric space into a separable metric space is uniformly continuous.

A fuller discussion of BISH, RUSS, and INT is found in Chapters 1,3 , and 5 of Bridges-Richman.

Since BISH is consistent with INT, RUSS, and CLASS-in other words, any proof of a proposition within BISH also counts as a proof of that proposition in each of these three varieties of mathematics-we have the possibility of independence results relative to BISH. For example, the statement UC is independent of BISH, as is its negation, because UC is a theorem of INT but there exists counterexample to UC within RUSS [BridgesRichman, Chapter 3, (3.3)]. The following independence result is an immediate consequence of Corollary 2 and Theorem 6 below, and is the main result of this paper.

Theorem 1. Each of the following statements is independent of BISH.

(i) There exists a bounded, pointwise continuous map of $[0,1]$ into $\mathbf{R}$ that is not Lebesgue measurable.

(ii) If $\mu$ is a positive measure on a locally compact space, then every real-valued map defined on a full set is measurable with respect to $\mu$.

From now on, we shall work within BISH, extended to either RUSS or INT where necessary. For the reader's convenience, we begin by summarizing those aspects of constructive analysis and measure theory that we shall need for our discussion of Lebesgue measurability. Full details of those matters are found in Chapters 
1-4 and 6 of Bishop-Bridges; Demuth-Kučera contains a development of measure theory within RUSS.

A metric space $(X, \rho)$ is said to be

compact if it is totally bounded and complete;

locally compact if each bounded set in $X$ is contained in a compact set.

Note that, following Bishop, we require totally bounded sets to be nonvoid; so that a compact or locally compact subset $S$ of a metric space $(X, \rho)$ is located, in the sense that the distance

$$
\rho(x, S) \equiv \inf \{\rho(x, y): y \in S\}
$$

from $x$ to $S$ exists for each $x$ in $X{ }^{3}$ The metric complement of a located set $S$ consists of all points $x$ in $X$ such that $\rho(x, S)>0$. A test function on a locally compact metric space $X$ is a uniformly continuous mapping $f: X \rightarrow \mathbf{R}$ such that $f(x)=0$ for all $x$ in the metric complement of some compact set (called a compact support of $f$ ); the set of test functions on $X$ is denoted by $C(X)$. A positive measure on $X$ is a nonzero linear mapping $\mu$ of $C(X)$ into $\mathbf{R}$ such that $\mu(f) \geq 0$ for each nonnegative test function $f$ on $X$.

To avoid excessive generality, we restrict attention to the integral constructed by extending a positive measure $\mu$ on a locally compact space $(X, \rho)$. A function $f: F \rightarrow \mathbf{R}$, where $F \subset X$, is said to be integrable if there exists a sequence $\left(f_{n}\right)$ of test functions on $X$ such that

$$
\begin{aligned}
& \sum_{n=1}^{\infty} \mu\left(\left|f_{n}\right|\right) \text { converges, and } \\
& \sum_{n=1}^{\infty} f_{n}(x)=f(x) \text { whenever } \sum_{n=1}^{\infty}\left|f_{n}(x)\right| \text { converges. }
\end{aligned}
$$

In that case, the sequence $\left(f_{n}\right)$ is called a representation of $f$ by test functions, and the integral of $f$,

$$
\int f d \mu \equiv \sum_{n=1}^{\infty} \mu\left(f_{n}\right)
$$

is independent of that representation; we also (loosely) call the function $f \mapsto \int f d \mu$ a positive measure on $X$. A subset $F$ of

\footnotetext{
${ }^{3}$ That is, there is an algorithm which, applied to a pair $(x, \varepsilon)$ with $x \in X$ and $\varepsilon>0$, computes $\rho(x, S)$ with an error at most $\varepsilon$.
} 
$X$ is full with respect to $\mu$ if there exists a $\mu$-integrable function whose domain includes $F$.

A complemented set in $X$ is an ordered pair $A \equiv\left(A^{1}, A^{0}\right)$ of subsets of $X$ such that $\rho(x, y)>0$ for all $x$ in $A^{1}$ and $y$ in $A^{0}$; the characteristic function of $A$ is the map $\chi_{A}: A^{1} \cup A^{0} \rightarrow$ $\{0,1\}$ such that $\chi_{A}\left(A^{1}\right)=\{1\}$ and $\chi_{A}\left(A^{0}\right)=\{0\}$. Operations on complemented sets $A$ and $B$ are defined in terms of their characteristic functions; for example, $A-B$ has characteristic function $\chi_{A}\left(1-\chi_{B}\right)$. An integrable set (with respect to $\mu$ ) is a complemented set $A$ whose characteristic function is integrable; $A$ then has measure $\mu(A) \equiv \int \chi_{A} d \mu$. If $f$ is an integrable function, then the complemented set

$$
(f \geq t) \equiv(\{x: f(x) \geq t\},\{x: f(x)<t\})
$$

is integrable for all but countably many $t>0$ [Bishop-Bridges, Chapter 6, (4.11)]. Note that $(f \geq t)$ is a complemented set, as all functions $f$ considered in integration theory are strongly extensional: that is, if $|f(x)-f(y)|>0$, then $\rho(x, y)>0$.

It can be shown that if $F$ is a full set and $A$ is an integrable set with positive measure, then $F \cap A^{1}$ is nonvoid [Bishop-Bridges, Chapter 6, (3.4)]; in particular, this means that, given an integrable set $A$ with positive measure, we can construct an element of $A^{1}$.

A compact integrable set is an integrable set $K \equiv\left(K^{1}, K^{0}\right)$ such that $K^{1}$ is compact and $K^{0}$ is the metric complement of $K^{1}$ in $X$; we usually then identify $K$ with $K^{1}$ and $X-K$ with $K^{0}$. A comparatively deep theorem of constructive measure theory states that if $A$ is an integrable set with positive measure, then for each $\varepsilon>0$ there exists a compact integrable set $K$ such that $K \subset A^{1}$ and $\mu(A-K)<\varepsilon$ [Bishop-Bridges, Chapter 6, (6.7)]. The following lemma, whose proof is included for completeness, depends on that result.

Lemma 1. Let $f$ be an integrable function, and $A$ an integrable set with positive measure, with respect to a positive measure $\mu$ on a locally compact space $X$. Then for each $\varepsilon>0$ there exist a compact integrable set $K$ and a test function $g: X \rightarrow \mathbf{R}$ such that $K \subset A^{1}, \mu(A-K)<\varepsilon$, and $|f(x)-g(x)|<\varepsilon$ for all $x$ in $K$.

Proof. Given $\varepsilon>0$, we may assume that $\mu(A)>\varepsilon$. By BishopBridges [Chapter 7, (3.13)], there exists a test function $g$ on $X$ such that $\int|f-g| d \mu<\varepsilon^{2} / 4$. Choose $\delta$ in $(\varepsilon / 2, \varepsilon)$ such that the complemented set $(|f-g| \geq \delta)$ is integrable, and let $B$ be 
the complemented set $A-(|f-g| \geq \delta)$. Then $B$ is integrable, $B^{1} \subset A^{1}$, and, as $\delta^{-1}|f(x)-g(x)| \geq 1$ whenever $\chi_{A-B}(x)=1$,

$$
\mu(A-B) \leq \delta^{-1} \int|f-g| d \mu<\varepsilon / 2 .
$$

Also, $|f(x)-g(x)|<\delta<\varepsilon$ for all $x$ in $B^{1}$. As $\mu(A)>\varepsilon>$ $\mu(A-B)$, we see that $\mu(B)>0$. We can therefore construct a compact integrable set $K$ such that $K \subset B^{1}$ and $\mu(B-K)<\varepsilon / 2$. Then $|f(x)-g(x)|<\varepsilon$ for all $x$ in $K$, and $K \subset A^{1}$; also,

$$
\mu(A-K) \leq \mu(A-B)+\mu(B-K)<\varepsilon .
$$

Let $\mu$ be a positive measure on a locally compact space $X$. A real-valued function $f$ defined on a full set is said to be measurable if for each integrable set $A$ and each $\varepsilon>0$, there exist an integrable set $B$ and an integrable function $g$ such that $B^{1} \subset A^{1}$, $\mu(A-B)<\varepsilon$, and $|f(x)-g(x)|<\varepsilon$ for all $x$ in $B^{1}$. If $f$ is a measurable function, and $g$ is an integrable function such that $|f(x)| \leq g(x)$ for all $x$ in a full set, then $f$ is integrable [BishopBridges, Chapter 6, (7.11)].

We now introduce a remarkable notion that plays an important role in the production of counterexamples within RUSS. Let $I$ denote the interval $[0,1]$, and let $0<\alpha<1$. A sequence $\left[a_{0}, b_{0}\right],\left[a_{1}, b_{1}\right], \ldots$ of proper closed subintervals of $I$ is called an $\alpha$-singular covering of $I$ if the following conditions hold:

(i) for all $m$ and $n$, either $\left[a_{m}, b_{m}\right]$ and $\left[a_{n}, b_{n}\right]$ are disjoint or they have at most an endpoint in common;

(ii) $a_{0}=0$ and $b_{1}=1$;

(iii) for each $x \in(0,1)$ there exist $m, n$ such that $b_{m}=a_{n}$ and $a_{m}<x<b_{n}$

(iv) the partial sums of the series $\sum_{n=0}^{\infty}\left|I_{n}\right|$ are bounded by $\alpha$.

It follows from (ii) and (iii) that for each $x$ in $I, 0 \leq x<b_{0}$, or $a_{1}<x \leq 1$, or there exist $m, n$ such that $b_{m}=a_{n}$ and $a_{m} \leq x \leq b_{n}$. (We remind the reader that, although the classical law of trichotomy does not hold in constructive mathematics, we can prove that if $a, b$ are real numbers such that $a<b$, then for each real number $x$ either $a<x$ or $x<b$.) 
We had better make it clear at this stage that our discussion of singular coverings is not vacuous: it is a theorem of RUSS that if $0<\alpha<1$, then there exists an $\alpha$-singular covering of $I \equiv[0,1]$ (cf. Beeson, Chapter 4, 6.1). ${ }^{4}$

If $^{5}\left(I_{n}\right)$ is an $\alpha$-singular covering of $I$ for some $\alpha$ with $0<$ $\alpha<1$, then the series $\sum_{n=0}^{\infty}\left|I_{n}\right|$ does not converge constructively. Classically, it converges to a nonrecursive real number.

Lemma 2. If $\left(I_{n}\right)$ is an $\alpha$-singular covering of $[0,1]$, where $0<$ $\alpha<1$, then $\left|I_{n}\right| \rightarrow 0$ as $n \rightarrow \infty$.

Proof. Let $I_{n} \equiv\left[a_{n}, b_{n}\right]$, as in the above definition. Given a positive integer $k$, for each $i \in\{1, \ldots, k-1\}$ choose $m_{i}, n_{i}$ such that $b_{m_{i}}=a_{n_{i}}$ and $a_{m_{i}}<i / k<b_{n_{i}}$; then there exists $\delta>0$ such that $a_{m_{i}}<y<b_{n_{i}}$ whenever $1 \leq i \leq k-1$ and $|y-i / k|<\delta$. Let

$$
N \equiv \max \left\{m_{1}, n_{1}, m_{2}, n_{2}, \ldots, m_{k-1}, n_{k-1}\right\},
$$

and consider any integer $n>N$. Property (i) of a singular covering ensures that $\rho\left(i / k, I_{n}\right) \geq \delta$ for $i=1, \ldots, k-1$; hence, for each such $i$, either $a_{n}>i / k$ or $a_{n}<i / k$. It follows that $0 \leq a_{n}<1 / k$, or $i / k<a_{n}<(i+1) / k$ for some $i$ with $1 \leq i \leq k-2$, or $a_{n}>(k-1) / k$. In the first case, as $\rho\left(1 / k, I_{n}\right)>$ 0 , we must have $I_{n} \subset[0,1 / k)$; similarly, in the second case $I_{n} \subset(i / k,(i+1) / k)$; and in the third, $I_{n} \subset((k-1) / k, 1]$. So in all cases, $\left|I_{n}\right|<1 / k$.

Given an $\alpha$-singular covering $\left(\left[a_{n}, b_{n}\right]\right)$ of $I \equiv[0,1]$, where $0<\alpha<1$, and a sequence $\left(c_{n}\right)$ of real numbers, we construct an associated canonical function $f: I \rightarrow \mathbf{R}$ as follows. For each natural number $n$, let $f_{n}$ be the uniformly continuous map of $\left[a_{n}, b_{n}\right]$ onto $I$ such that

$$
\begin{aligned}
& f_{n}(x)=0 \text { if } \rho\left(x,\left[a_{n}, b_{n}\right]\right)>0, \\
& f_{n}\left(\frac{1}{2}\left(a_{n}+b_{n}\right)\right)=1 \text {, and } \\
& f_{n} \text { is linear on each half of }\left[a_{n}, b_{n}\right] .
\end{aligned}
$$

We claim that $f \equiv \sum_{n=0}^{\infty} c_{n} f_{n}$ is a well-defined function on $I$, and that it is pointwise continuous on $I$. To see this, consider any $x$ in $I$. If $x<b_{0}$, then, by properties (i) and (ii) of a

${ }^{4}$ An $\alpha$-singular covering of the classical interval $[0,1]$ is clearly impossible; but there exist $\alpha$-singular coverings of the set of recursive real numbers in the classical interval $[0,1]$.

${ }^{5}$ Throughout this paper we shall use $\left(I_{n}\right)$ as a shorthand for $\left(I_{n}\right)_{n=0}^{\infty}$. 
singular covering, $f_{n}(y)=0$ for all $n \geq 1$ and all $y \in I$ such that $|x-y|<b_{0}-x$; so $f(y)=c_{0} f_{0}(y)$ for all such $y$; whence $f$ is pointwise continuous at $x$. If $0<x<1$, then, choosing $M, N$ such that $b_{M}=a_{N}$ and $a_{M}<x<b_{N}$, we see from property (i) of a singular covering that $f_{n}(x)=0$ for all $n$ other than $M$ and $N$; then $f(y)=c_{M} f_{M}(y)+c_{N} f_{N}(y)$ for all $y$ in $\left(a_{M}, b_{N}\right)$; so again $f$ is defined and pointwise continuous at $x$. Finally, the case $a_{1}<x$ is handled similarly to the case $x<b_{0}$.

Note that $f(x)=c_{n} f_{n}(x)$ for any $x$ in $\left[a_{n}, b_{n}\right]$; that $f\left(a_{n}\right)=$ $f\left(b_{n}\right)=f_{n}\left(a_{n}\right)=f_{n}\left(b_{n}\right)=0$; and hence that if the sequence $\left(c_{n}\right)$ is bounded, then so is the function $f$.

Theorem 2. Let $\left(I_{n}\right)$ be an $\alpha$-singular covering of $[0,1]$, where $0<\alpha<1$, and let $\left(c_{n}\right)$ be a sequence of real numbers. Then the canonical function associated with $\left(I_{n}\right)$ and $\left(c_{n}\right)$ is Lebesgue integrable if and only if the series $\sum_{n=0}^{\infty}\left|c_{n}\right|\left|I_{n}\right|$ is convergent.

Proof. Let $f$ be the canonical function in question. As we observed above, $f$ is pointwise continuous. Suppose that $f$ is Lebesgue integrable; then $|f|$ is Lebesgue integrable. So, given $\varepsilon$ with $0<\varepsilon \leq 1 / 8$, we can use Bishop-Bridges (Chapter 7, (3.13)) to construct a uniformly continuous function $h$ on $I$ such that $\int|(|f|-h)| d \mu<\varepsilon$. Choose a positive integer $k$ such that $|h|<k$ on $I$; then for any finite set $D$ of natural numbers,

$$
\sum_{n \in D} \int|f| \chi_{I_{n}} d \mu<\varepsilon+\sum_{n \in D} k\left|I_{n}\right|
$$

Now let $p$ be a positive integer such that $2 / p<\varepsilon$. By Lemma 1 , we can find a compact integrable set $K$ and a uniformly continuous function $g$ on $I$ such that $K \subset I, \mu(I-K)<\varepsilon / k$, and ||$f|-g|<\varepsilon / p$ on $K$. Let $\delta>0$ be such that $|g(x)-g(y)|<$ $1 / 16 p$ whenever $x, y \in I$ and $|x-y|<\delta$. By Lemma 2, there exists $N$ such that $\left|I_{n}\right|<\delta / 2$ for all $n \geq N$. Consider any $n \geq N$ for which $\left|c_{n}\right| \geq 1 / p$ and $I_{n} \cap K$ is nonvoid, and let $x \in I_{n} \cap K$. Either $|f(x)|>7\left|c_{n}\right| / 16$ or $|f(x)|<9\left|c_{n}\right| / 16$. In the first case, let $y$ be a point of $I_{n}$ such that $|f(y)|<\left|c_{n}\right| / 16$, choose $\delta^{\prime}>0$ so that ||$f(z)|-| f(y)||<\left|c_{n}\right| / 16$ whenever $z \in I$ and $|z-y|<\delta^{\prime}$, and suppose that there exists $z \in K$ such that $|z-y|<\min \left\{\frac{1}{2} \delta, \delta^{\prime}\right\}$. Then as $|z-x| \leq|z-y|+\left|I_{n}\right|<\delta$, we 
have

$$
\begin{aligned}
|| f(x)|-| f(y)|| & \leq|| f(x)|-g(x)|+|g(x)-g(z)| \\
& +|g(z)-| f(z)||+|| f(z)|-| f(y)|| \\
& <\left|c_{n}\right|(\varepsilon+1 / 16+\varepsilon+1 / 16) \\
& <3\left|c_{n}\right| / 8 \\
& <|| f(x)|-| f(y)||,
\end{aligned}
$$

a contradiction. Hence $|y-z| \geq \min \left\{\frac{1}{2} \delta, \delta^{\prime}\right\}$ for all $z$ in $K$, so $y \in I-K$. It follows from elementary geometry, with reference to the definition of the function $f$, that $I_{n}-K$ contains a union of two disjoint intervals, each of length $\left|I_{n}\right| / 32$; see Figure (a) below. Similarly, if $|f(x)|<9\left|c_{n}\right| / 16$, then $I_{n}-K$ contains an interval of length $\left|I_{n}\right| / 16$; see Figure (b).

Now, for any $n \geq N$ such that $\left|c_{n}\right| \geq 1 / p$, either $\left|I_{n}\right| / 16>$ $\mu\left(I_{n}-K\right)$ or $\mu\left(I_{n}-K\right)>\left|I_{n}\right| / 32$. In the former case, $I_{n} \cap K$ must be empty, by the foregoing, so $\mu\left(I_{n}-K\right)=\left|I_{n}\right|>\left|I_{n}\right| / 16-a$

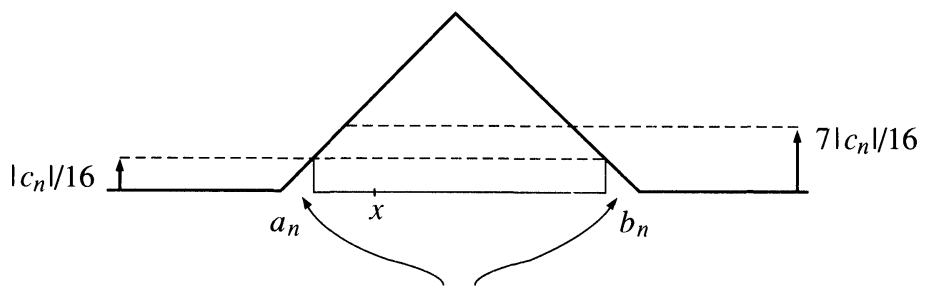

Two disjoint intervals, each of length $\left|I_{n}\right| / 32$, contained in $I_{n}-K$

Figure (a). $x \in I_{n} \cap K,|f(x)|>7\left|c_{n}\right| / 16$.

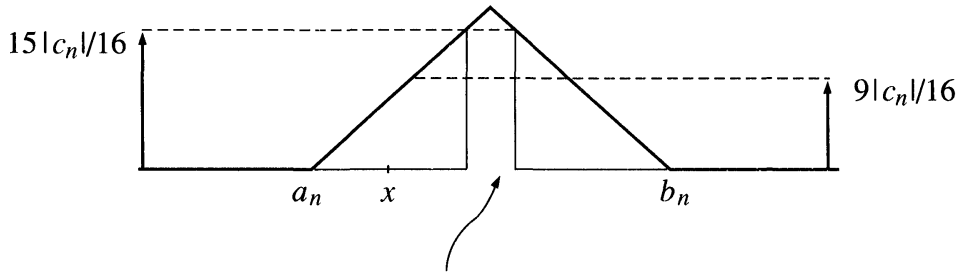

An interval of length $\left|I_{n}\right| / 16$, contained in $I_{n}-K$

FiguRE (b). $x \in I_{n} \cap K,|f(x)|<9\left|c_{n}\right| / 16$. 
contradiction. Thus if $D$ is a finite set of natural numbers such that $n \geq N$ and $\left|c_{n}\right| \geq 1 / p$ for each $n \in D$, then

$$
\sum_{n \in D}\left|I_{n}\right|<32 \sum_{n \in D} \mu\left(I_{n}-K\right) \leq 32 \mu(I-K)<32 \varepsilon / k,
$$

and therefore, by $(*)$,

$$
\sum_{n \in D} \frac{1}{2}\left|c_{n}\right|\left|I_{n}\right|=\sum_{n \in D} \int|f| \chi_{I_{n}} d \mu<\varepsilon+32 \varepsilon=33 \varepsilon .
$$

Now consider integers $j>i \geq N$. Partition the set $\{i+1, i+$ $2, \ldots, j\}$ into disjoint subsets $D, E$ such that $\left|c_{n}\right|>1 / p$ if $n \in$ $D$, and $\left|c_{n}\right|<2 / p$ if $n \in E$. Then

$$
\begin{aligned}
\sum_{n=i+1}^{j}\left|c_{n}\right|\left|I_{n}\right| & =\sum_{n \in D}\left|c_{n}\right|\left|I_{n}\right|+\sum_{n \in E}\left|c_{n}\right|\left|I_{n}\right| \\
& \leq 2(33 \varepsilon)+(2 / p) \sum_{n \in E}\left|I_{n}\right| \\
& \leq 66 \varepsilon+\varepsilon \alpha \\
& <67 \varepsilon .
\end{aligned}
$$

As $\varepsilon$ is arbitrary, it follows that the sequence of partial sums of the series $\sum_{n=0}^{\infty}\left|c_{n}\right|\left|I_{n}\right|$ is a Cauchy sequence; whence that series converges.

Conversely, suppose that the series $\sum_{n=0}^{\infty}\left|c_{n}\right|\left|I_{n}\right|$ converges. Then the series

$$
\sum_{n=0}^{\infty} \int\left|c_{n} f_{n}\right| d \mu=\frac{1}{2} \sum_{n=0}^{\infty}\left|c_{n}\right|\left|I_{n}\right|
$$

converges; so, by the definition of "integrable function," $f \equiv$ $\sum_{n=0}^{\infty} c_{n} f_{n}$ is Lebesgue integrable.

A sequence $\left(c_{n}\right)$ of real numbers is eventually bounded away from 0 if there exist $\delta>0$ and a positive integer $N$ such that $\left|c_{n}\right|>\delta$ for all $n \geq N$.

Corollary 1. Let $\left(I_{n}\right)$ be an $\alpha$-singular covering, where $0<\alpha<$ 1 , and let $\left(c_{n}\right)$ be a bounded sequence of real numbers that is eventually bounded away from 0 . Then the canonical function associated with $\left(I_{n}\right)$ and $\left(c_{n}\right)$ is bounded, pointwise continuous, but not Lebesgue measurable.

Proof. We have already shown that $f$ is bounded and pointwise continuous. Suppose it is Lebesgue measurable and therefore, being bounded, Lebesgue integrable. Then, by Theorem 2 , the series $\sum_{n=0}^{\infty}\left|c_{n}\right|\left|I_{n}\right|$ is convergent. Choose $\delta>0$ and a positive integer 
$N$ such that $\left|c_{n}\right|>\delta$ for all $n \geq N$. Then as $\left|I_{n}\right| \leq \delta^{-1}\left|c_{n}\right|\left|I_{n}\right|$ for all such $n$, the series $\sum_{n=0}^{\infty}\left|I_{n}\right|$ converges, which is absurd.

Corollary 2. In RUSS there exist functions $g, G$ on $I \equiv[0,1]$ such that

(i) $g$ is bounded, pointwise continuous, and not Lebesgue measurable;

(ii) $G$ is pointwise differentiable and satisfies a Lipschitz condition;

(iii) $G^{\prime}=g$.

Proof. Let $\left(I_{n}\right)$ be an $\alpha$-singular covering of $I \equiv[0,1]$, where $0<\alpha<1$, and set

$$
J_{0} \equiv I_{0}, \quad J_{1} \equiv I_{1}, \quad c_{0}=c_{1} \equiv 0 .
$$

For any integer $n \geq 2$, let $J_{2 n-2}$ and $J_{2 n-1}$ be the left and right halves of $I_{n}$, respectively, and let $c_{2 n-2}=-c_{2 n-1} \equiv 1$. It is easy to show that $\left(J_{m}\right)_{m=0}^{\infty}$ is an $\alpha$-singular covering of $[0,1]$. As $\left(c_{n}\right)$ is bounded, the canonical function $g$ associated with $\left(J_{m}\right)$ and $\left(c_{m}\right)$ is bounded; also, by Corollary $1, g$ is pointwise continuous but not Lebesgue measurable. For each $n, g$ is uniformly continuous on $I_{n}$ and satisfies $\int \chi_{I_{n}} g d \mu=0$. Consequently, for each $n$, the function $G_{n}$, defined by

$$
\begin{aligned}
G_{n}(x) & =0 & & \text { if } x \in I \text { and } \rho\left(x, I_{n}\right)>0, \\
& =\int_{a_{n}}^{x} g & & \text { if } x \in I_{n}=\left[a_{n}, b_{n}\right],
\end{aligned}
$$

is uniformly continuous and pointwise differentiable on $I$; also, for each $x \in I, G_{n}^{\prime}(x)=g(x)$ if $x \in I_{n}$, and $G_{n}^{\prime}(x)=G_{n}(x)=0$ if $\rho\left(x, I_{n}\right)>0$. An argument similar to that used before Theorem 2 , in our discussion of canonical functions, now shows that $G \equiv$ $\sum_{n=0}^{\infty} G_{n}$ is pointwise differentiable, that $G^{\prime}=g$ on $I$, and that $G=G_{n}$ on $I_{n}$. As $G$ is uniformly continuous on $I$, to prove that it satisfies a Lipschitz condition it will suffice to show that if $x \in I_{m} \equiv\left[a_{m}, b_{m}\right]$ and $y \in I_{n} \equiv\left[a_{n}, b_{n}\right]$, where $m \leq n$, then $|G(y)-G(x)| \leq|y-x|$. If $m=n$, then, as $|g| \leq 1$,

$$
|G(y)-G(x)|=\left|\int_{a_{m}}^{y} g-\int_{a_{m}}^{x} g\right|=\left|\int_{x}^{y} g\right| \leq|y-x| .
$$

If $m<n$, then, as $G\left(a_{n}\right)=G\left(b_{m}\right)=0$, we have

$$
\begin{aligned}
|G(y)-G(x)| & \leq\left|G(y)-G\left(a_{n}\right)\right|+\left|G\left(b_{m}\right)-G(x)\right| \\
& \leq\left|y-a_{n}\right|+\left(a_{n}-b_{m}\right)+\left|b_{m}-x\right| \\
& =y-a_{n}+a_{n}-b_{m}+b_{m}-x \\
& =y-x=|y-x| . \quad \square
\end{aligned}
$$


Theorem 2 first appeared in Demuth's unpublished doctoral thesis [Demuth 1964], and was rediscovered recently by Bridges. Note that our construction of a bounded, pointwise continuous function that is not Lebesgue measurable, and our proof that the function has that property, are simpler than their counterparts in Kushner (Chapter 8, Theorem 10), which seems likely to become the standard reference for RUSS in the west.

In this context it is appropriate to mention, without proof, some related results in RUSS:

Theorem 3. In RUSS, there exists a function which satisfies a Lipschitz condition on $[0,1]$, but is not differentiable at any point of [0,1] [Demuth 1969a].

A function $f$ on a closed interval $[a, b]$ in $\mathbf{R}$ is absolutely continuous if for each natural number $n$ there exists a piecewise linear function $p_{n}$ such that

$$
\sum_{k=0}^{m-1}\left|\left(f-p_{n}\right)\left(x_{k+1}\right)-\left(f-p_{n}\right)\left(x_{k}\right)\right| \leq 2^{-n}
$$

whenever $a \leq x_{0} \leq x_{1} \leq \cdots \leq x_{m} \leq b$.

Theorem 4. In RUSS, if $F$ is an absolutely continuous function on $[0,1]$, then there exists a full set $A$ such that $F$ is differentiable at any point of $A^{1} \cap(0,1)$ [Demuth 1968].

Theorem 5. In RUSS, the following are equivalent conditions on a function $f$ defined on $[0,1]$ :

(i) $f$ is Lebesgue integrable;

(ii) there is an absolutely continuous, pointwise differentiable function $F$ on $[0,1]$, such that $F^{\prime}(x)=f(x)$ for each $x$ in $(0,1)$ [Demuth 1965];

(iii) there exist an absolutely continuous function $F$, and $a$ full subset $A$ of $[0,1]$, such that for each $x$ in $A^{1} \cap$ $(0,1), F$ is differentiable at $x$ and $F^{\prime}(x)=f(x)$ [Demuth 1969b].

We turn, finally, to Lebesgue measurability within INT. In the case of Lebesgue measure on $\mathbf{R}^{n}$, our next theorem is found in Heyting [6.2.2, Theorem 1]; our proof is neater and more general than Heyting's. 
Theorem 6. In INT, if $\mu$ is a positive measure on a locally compact space $X$, then every real-valued map defined on a full subset of $X$ is measurable with respect to $\mu$.

Proof. It will suffice to prove that if $F$ is a full subset of $X, f$ a mapping of $F$ into $\mathbf{R}, A$ an integrable set with positive measure, and $\varepsilon>0$, then there exist an integrable set $K$ and a test function $g$ such that $K^{1} \subset A^{1}, \mu(A-K)<\varepsilon$, and $f(x)=g(x)$ for all $x$ in $K^{1}$. To this end, note that $B \equiv\left(A^{1} \cap F, A^{0} \cap F\right)$ is an integrable set, with $\mu(B)=\mu(A)$. Construct a compact integrable set $K$ such that $K^{1} \subset B^{1} \subset A^{1}$ and $\mu(A-K)=\mu(B-K)<\varepsilon$. In view of $\mathrm{UC}$, we see that $f$ is uniformly continuous on $K^{1}$. To complete the proof, we need only use the Tietze Extension Theorem [Bishop-Bridges, Chapter 4, (6.16)] to construct a test function $g: X \rightarrow \mathbf{R}$ such that $f(x)=g(x)$ for all $x$ in $K^{1}$.

It follows from Corollary 2 and Theorem 6 that, as was stated more formally in Theorem 1, we can neither prove nor disprove, within BISH alone, that every bounded, pointwise continuous function on $[0,1]$ is Lebesgue measurable.

Finally, we refer the interested reader to pp. 63-64 of BridgesRichman, for the construction, within RUSS, of a compact subset of $[0,1]$ that is not Lebesgue measurable.

\section{REFERENCES}

[Beeson] M. J. Beeson, Foundations of constructive mathematics, Ergeb. Math. Grenzgeb., Folge 3, Band 6, Springer-Verlag, Berlin and New York, 1985.

[Bishop-Bridges] Errett Bishop and Douglas Bridges, Constructive analysis, Grundlehren Math. Wiss., Band 279, Springer-Verlag, Berlin and New York, 1985.

[Bridges-Richman] Douglas Bridges and Fred Richman, Varieties of constructive mathematics, London Math. Soc. Lecture Notes, no. 97, Cambridge Univ. Press, 1987.

[Demuth 1964] O. Demuth, On Lebesgue integration in constructive analysis, Doctoral thesis, Moscow State Univ., 1964. (Russian)

[Demuth 1965] __, On Lebesgue integration in constructive analysis, Dokl. Akad. Nauk USSR 160 (6), 1239-1241, 1965. (Russian)

[Demuth 1967] __, The Lebesgue integral in constructive analysis, Zapisky Nauk. Sem. Leningrad Otdel. Mat. Inst. Steklov. 4 (1967), 30-43. (Russian)

[Demuth 1968] _ The Lebesgue integral and the concept of function measurability in constructive analysis, Zapisky Nauk. Sem. Leningrad Otdel. Mat. Inst. Steklov. 8 (1968), 21-28. (Russian)

[Demuth 1969a] __, On the differentiability of constructive functions, Comment. Math. Univ. Carolinae 10 (1969), 167-175. (Russian) 
[Demuth 1969b] _ The spaces $L_{r}$ and $S$ in constructive mathematics, Comment. Math. Univ. Carolinae 10 (1969), 261-284. (Russian)

[Demuth-Kučera] O. Demuth and A. Kučera, Remarks on constructive mathematical analysis, Logic Colloquium '78 (M. Boffa, D. van Dalen, and K. McAloon, eds.), North-Holland, Amsterdam, 1979, pp. 81-129.

[Heyting] A. Heyting, Intuitionism-an introduction, 3rd ed., North-Holland, Amsterdam, 1971.

[Kfoury et al.] A. J. Kfoury, Robert N. Moll, and Michael A. Arbib, A programming approach to computability, Springer-Verlag, Berlin and New York, 1982.

[Kushner] B. A. Kushner, Lectures on constructive mathematical analysis, Math. Monographs, vol. 60, Amer. Math. Soc. Providence, RI, 1985.

[Rogers] Hartley Rogers, Theory of recursive functions and effective computability, McGraw-Hill, New York, 1967.

Department of Mathematics and Statistics, University of Waikato, Private Bag 3105, Hamilton, New Zealand

MFF UK, Malostranské nåm. 2, 11000 Praha 1, Czechoslovakia 